\title{
Synthesis and Surface Modification of Calcium Carbonate Nanoparticles Using Ultrasound Cavitation Technique
}

\author{
Navinchandra Shimpi ${ }^{1, *}$, Ananda Mali ${ }^{2}$, D.P. Hansora ${ }^{2}$, Satyendra Mishra ${ }^{2}$ \\ ${ }^{1}$ Department of Chemistry, University of Mumbai, Kalina, Santacruz East, Mumbai, MS, India \\ ${ }^{2}$ University Institute of Chemical Technology, North Maharashtra University, Jalgaon 425 001, MS, India
}

Copyright (C) 2015 Horizon Research Publishing All rights reserved.

\begin{abstract}
Calcium carbonate nanoparticles $\left(\mathrm{nCaCO}_{3}\right)$ was synthesized using optimized process of ultrasound assisted precipitation technique. Due to ultra-sonication phenomena, reacting components of reaction mixture gets dispersed uniformly, and at the same time $\mathrm{nCaCO}_{3}$ particles agglomerate with each other due to strong electrostatic force of attraction developed over their surface. The de-agglomeration and charge nullification were achieved using surface modification of $\mathrm{CaCO}_{3}$ nanoparticles using triethoxy vinyl silane (TEVS) under ultrasound environment. The surface modification of $\mathrm{nCaCO}_{3}$ particles was confirmed using Fourier transforms infrared (FTIR) spectroscopy. Size, shape and formation of $\mathrm{nCaCO}_{3}$ particles at nanoscale were studied using transmission electron microscopy (TEM). The size of unmodified and modified $\mathrm{nCaCO}_{3}$ was found to be $\approx 50-80$ and $50-90 \mathrm{~nm}$, respectively, with square crystal structure morphology. Due to the surface modification by TEVS, thin layer was formed over the surface of $\mathrm{nCaCO}_{3}$ particle, which was confirmed by TEM micrograph. The purpose behind carry out this reaction under ultrasound environment is to optimize synthesis procedure of $\mathrm{nCaCO}_{3}$ particles, to provide effective ultrafine precipitation and give stable \& uniform dispersion of inorganic particles with reactants. Moreover, the acoustic waves of ultrasound treatment are an effective controlling parameter to obtain uniform dispersion of inorganic nanoparticles in solution by producing non-linear effects in reaction mixture. In this ultrasound treatment, transient cavitation and acoustic stretching are the most dominant affecting parameters, which give homogeneous dispersion of $\mathrm{nCaCO}_{3}$.
\end{abstract}

Keywords Ultra-sonication, Surface Modified $\mathrm{nCaCO}_{3}$, TEM, and FTIR

\section{Introduction}

Various research group have successfully incorporated inorganic nanofillers in to the polymers like polypropylene (PP) [1-4], epoxy [5-7], polyamide (PA) [8], polyvinyl chloride (PVC) [9-11], polystyrene (HIPS) [12-13], poly methyl methacrylate (PMMA) [14], and rubber like styrene butadiene rubber (SBR) $[3,10,15]$, silicone rubber $[16,17]$, Ethylene propylene diene monomer rubber(EPDM) [18] and polybutadiene rubber (PBR) [19] for improvement in their thermal, mechanical and rheological. Inorganic nanoparticles have received an acceptance as reinforcing \& hydrophobic fillers, because of their ease of fabrication and low cost.

$\mathrm{CaCO}_{3}$ is one of the most important inorganic filler which can be used in plastics, rubber and paint industries but it's hydrophilic nature creates the problem of dispersion in polymer matrix. The elegant approaches like insitu deposition [1,5,8,10-11], conventional precipitation method $[1,20]$, solution spray process $[2,16-17,19]$, matrix mediated growth technique $[3,9,15]$, solution bubbling \& industrial carbonation methods [11], crystallization and sonochemical carbonization [13-14] and ultrasound cavitation [7]have been used for the synthesis of $\mathrm{nCaCO}_{3}$ particles. Surface modification of $\mathrm{CaCO}_{3}$ with hydrophobic speciescan solve this problem, which also lead them for major applications as mineral particles reinforced polymer nanocomposites [1,4,11,16-17,21-22]. The surface modification using various surfactants has been extensively studied e.g. bio and synthetic molecules [23-24], the surfactants containing reactive functional groups such as silane coupling agents $[2,4,22]$, titanate coupling agents, biomacromolecules, designed peptide, double hydrophobic block copolymers, micelles, vesicles, sodium stearate, dihydrogen phosphate and oleic acid [11], stearic acid [6,16-17,20], and sodium oleate [13]which can improve the hydrophobic nature of $\mathrm{CaCO}_{3}$. Above all, surfactants have been studied to elucidate their effect on crystallization of $\mathrm{CaCO}_{3}$ particles. Because of high hydrophobic character, the surfaces can possess interesting characteristics, like low water and snow adhesion, low friction in surface dragging and easy dirt removal [22].

Particle size and morphology of $\mathrm{CaCO}_{3}$ particles heavily depend on preparation methods. Inorganic nanoparticles have tendency to agglomerate because of their high surface energy, so it is difficult to achieve the homogeneous dispersion of these nanoparticles in polymer matrix [11]. 
Also, high density of inorganic particles allows them to settle down or to form aggregates. Surface modification of $\mathrm{nCaCO}_{3}$ by ultrasound cavitation technique is the best way to reduce their surface energy up to some extent and also increase their compatibility with polymer matrix along with homogeneity into the bonding. The surface of the $\mathrm{CaCO}_{3}$ nanoparticles must be hydrophobic that is required for encapsulation and close interaction with the polymer chains [6-7,13,18-19,23], which also depend on the functional groups of surfactants to be modified over the surface of $\mathrm{CaCO}_{3}$ nanoparticles. Therefore, the $\mathrm{CaCO}_{3}$ nanoparticles were treated by a reactive silane coupling agent triethoxy vinyl silane (TEVS) to obtain the functionalized surface of $\mathrm{CaCO}_{3}$ nanoparticles. The reaction can proceed by reacting each $\mathrm{Si}-\mathrm{OC}_{2} \mathrm{H}_{5}$ groups with hydroxyl groups on the surface of nanoparticles and as a result surface of $\mathrm{CaCO}_{3}$ nanoparticles were modified by bondingTEVS molecules.

The main importance of this research work is to synthesize the surface modified $\mathrm{CaCO}_{3}$ nanoparticle using an ultrasound assisted precipitation technique by considering the need of the industry, which could be a continuous and an elegant approach for production. The present work also focuses the use of ultrasonic irradiations for uniform dispersion of agglomerated materials into the reacting mixture, which can control the effectiveness of overall synthesis and surface modification of $\mathrm{CaCO}_{3}$ nanoparticles. When ultrasonic waves pass through a liquid medium large numbers of micro bubbles form, grow and collapse in very short period of time, which lead to the cavitational effects on intense turbulence, liquid circulation currents and formation of free motion to the molecular level. In present study, the collapse of cavitation bubbles near the interface of immiscible liquids can cause disruption of the phase required for formation of very fine nanoparticles. And at the same time growth of electrostatic forces may cause agglomeration, which can be overcome by encapsulation or surface modification of nanoparticles.

\section{Experimental}

\subsection{Materials}

Calcium chloride $\left(\mathrm{CaCl}_{2}\right)$, ammonium bicarbonate $\left(\mathrm{NH}_{4} \mathrm{HCO}_{3}\right)$, sodium dodecyl sulphate (SDS), acetone and TEVS (modifying coupling agent) were procured from S. D. fine chemicals Ltd, Mumbai (India).

\subsection{Synthesis of $\mathrm{CaCO}_{3}$ Nanoparticles}

$0.5 \mathrm{M}$ solution of $\mathrm{NH}_{4} \mathrm{HCO}_{3}$ was mixed in a beaker containing solution of $250 \mathrm{ml}$ of distilled water and $0.7 \mathrm{~g}$ of SDS, which was dissolved properly and this solution was denoted as solution A. Similarly, in another beaker $0.25 \mathrm{M}$ solution of $\mathrm{CaCl}_{2}$ was dissolved in solution $(25 \mathrm{ml}$ of distilled water $+0.25 \mathrm{wt} \%$ of SDS) which was denoted as solution B. $1.25 \mathrm{~g}$ of SDS was dissolved in $500 \mathrm{ml}$ distilled water denoted as solution C. As shown in Figure 1, Ultra sonication probe was inserted in beaker containing solution $\mathrm{C}$ and sonication was started. After 5 min of ultrasound treatment, both solution of A and B were added by drop wise at the constant rate using burette, during this mixing ultrasound effect which was kept continue till complete addition of both solutions.

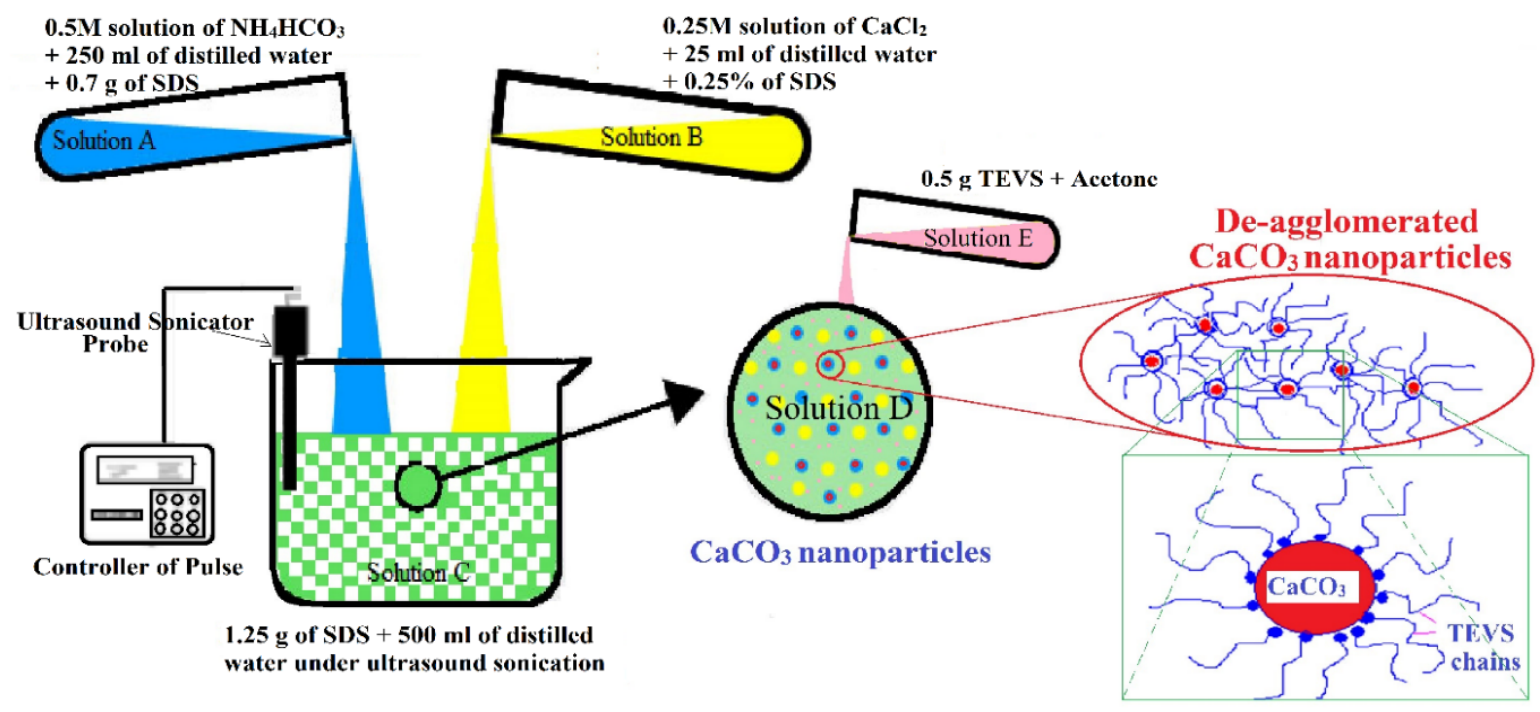

Figure 1. Schematic representation of reaction setup with ultrasonic probe for synthesis of $\mathrm{nCaCO}_{3}$ particles by ultrasound cavitation technique. 


\subsection{Surface Modification of $\mathrm{CaCO}_{3} \mathrm{Nanoparticles}$}

The various coupling agent (silane coupling agents e.g. TEVS)can be used effectively for surface modification of $\mathrm{CaCO}_{3}$ nanoparticles[2,4,11]. $100 \mathrm{~g}$ of $\mathrm{nCaCO}_{3}$ particles was uniformly dispersed in acetone under ultrasound environment for 30 min to form $\mathrm{CaCO}_{3}$ nanoparticles, this solution was denoted as D, while $5 \mathrm{~g}$ of TEVS was also dissolved in acetone and denoted as solution E (Figure 1). The ultrasonic probe was also immersed in solution D to generate sonic waves and at the same time solution $\mathrm{E}$ was added drop wise in solution $\mathrm{D}$ with constant rate. The effect of ultrasound cavitation provides very efficient mixing and strong dispersion. At the initial period of $\mathrm{nCaCO}_{3}$ particles preparation, these particles were agglomerated due development of strong electrostatic forces of attraction. This agglomeration was discarded by surface modification as discussed in earlier texts. Figure 2 shows the schematic representation of synthesis of $\mathrm{CaCO}_{3}$ nanoparticles without surface modification and with surface modification, both underultrasound environment. Without surface modified $\mathrm{CaCO}_{3}$ nanoparticles showed clusters or agglomerated forms, while surface modified one showed de-agglomerated $\mathrm{CaCO}_{3}$ nanoparticles.

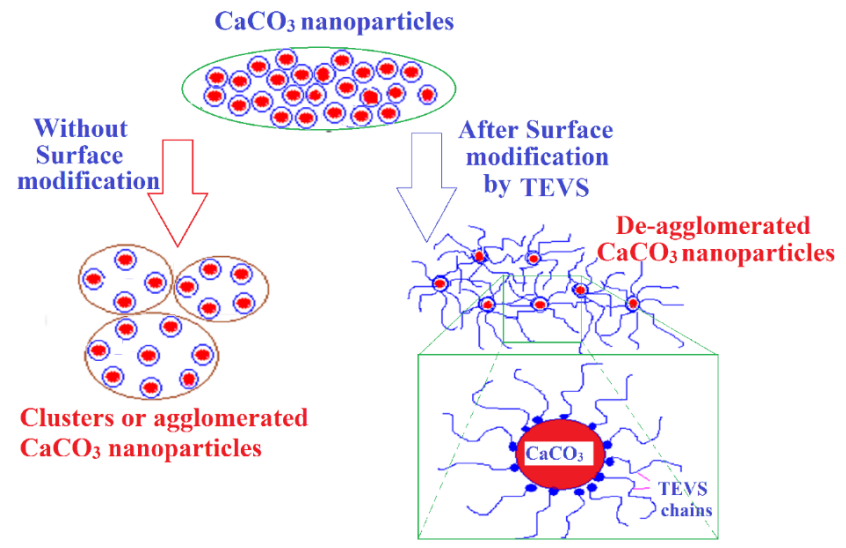

Figure 2. Schematic representation of surface modification of $\mathrm{nCaCO}_{3}$ particles by TEVS.

\subsection{Characterizations}

FTIR spectrophotometer (model- shimadzu, 8400, Tokyo, Japan) was used to obtain the IR spectra of TEVS, which can confirm the bond formation of TEVS with surface of $\mathrm{nCaCO}_{3}$ particles. Samples were finely divided and dispersed in a $\mathrm{KBr}$ powder for analysis. The scans were taken for each sample recorded for $4000-400 \mathrm{~cm}^{-1}$ at a resolution of $4 \mathrm{~cm}^{-1}$ in the transmittance mode. The morphology, exact size and shape of $\mathrm{nCaCO}_{3}$ particles were investigated by TEM (CM200, Philips Netherlands) were used at a resolution of $2.4 \AA$. The samples were dispersed in water and deposited on a copper grid before viewing under the microscope.

\section{Results and Discussions}

\subsection{Confirmation of Surface Modification Using FTIR}

FTIR absorption spectra of unmodified $\mathrm{nCaCO}_{3}$ particles and surface modified $\mathrm{nCaCO}_{3}$ particles are shown in Figure 3 (a-b). In the spectrum of unmodified $\mathrm{CaCO}_{3}$ nanoparticles denoted as (a) in Figure 3, the broad absorption band was observed at $3435 \mathrm{~cm}^{-1}$ which confirm the presence of hydroxyl $(\mathrm{OH})$ group on the surface of $\mathrm{nCaCO}_{3}$ particles due to presence of moisture[2].

The absorption that occurred around $2900 \mathrm{~cm}^{-1}$ and 2850 $\mathrm{cm}^{-1}$ was characteristic of $\mathrm{H}-\mathrm{C}-\mathrm{H}$ asymmetric and symmetric stretching vibrations [16-17,22]. TEVS surface modified $\mathrm{CaCO}_{3}$ nanoparticles showed the sharp peak at $1641 \mathrm{~cm}^{-1}$ which indicated unsaturated $\mathrm{C}=\mathrm{C}$ double bond of ethoxy group (present in TEVS)attached to surface of $\mathrm{CaCO}_{3}$ nanoparticles[2]. Silicone could be characterized by $\mathrm{Si}-\mathrm{CH}_{3}$ symmetric deformation, stretching vibration observed at 802 $\mathrm{cm}^{-1}$, while $\mathrm{Si}-\mathrm{O}-\mathrm{Si}$ asymmetric stretching vibration was observed in the range $1100-1090 \mathrm{~cm}^{-1}[2]$. From these spectra, it is clear that silicone was successfully attached to $\mathrm{CaCO}_{3}$ nanoparticles. The broad absorption bands as shown in (b) spectra of Figure 3 ranging between 1098 and $1082 \mathrm{~cm}^{-1}$ were characteristic peaks of Si-O-C group present in TEVS, which indicates the formation of $\mathrm{Si}-\mathrm{O}-\mathrm{CaCO}_{3}$ bond on surface modified $\mathrm{CaCO}_{3}$ nanoparticle[2,22]. Schematic representation of surface modification of $\mathrm{CaCO}_{3}$ nanoparticles is already shown in Figure 2.

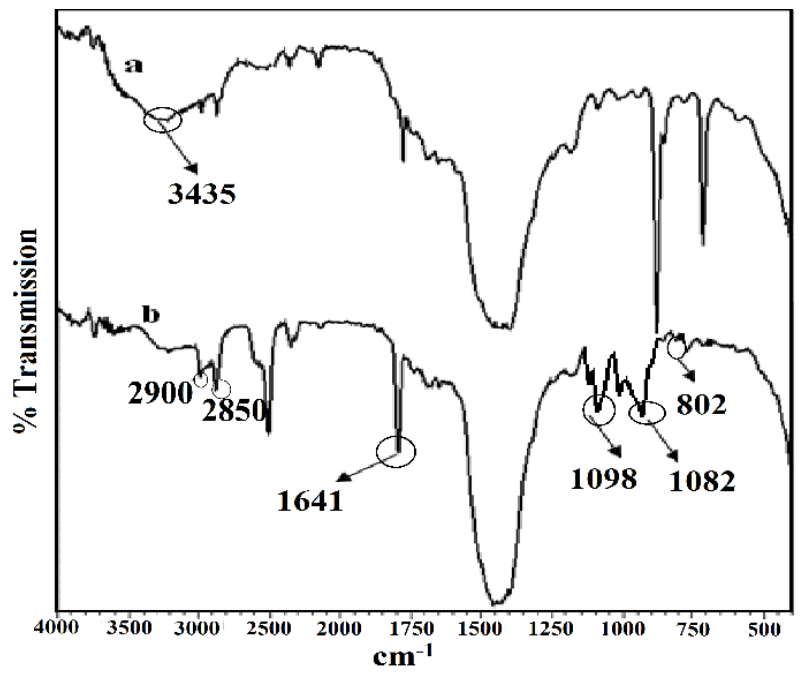

Figure 3. (a) FTIR spectra of surface modified $\mathrm{nCaCO}_{3}$ particles (b) Schematic representation of mechanism of surface modification of $\mathrm{nCaCO}_{3}$.

\subsection{Size and Shape of $\mathrm{CaCO}_{3}$ Nanoparticles}

The size of unmodified $\mathrm{CaCO}_{3}$ nanoparticles was recorded $\sim 50-80 \mathrm{~nm}$ with few agglomerations, and nature of particles was rod shape as shown in Figure 4(a). The size of TEVS modified $\mathrm{CaCO}_{3}$ nanoparticles was recorded in the range of $\sim 50-90$ nmas shown in Figure 4(b). Particle size of unmodified $\mathrm{nCaCO}_{3}$ particles was observed having size $<39$ $\mathrm{nm}$ and surface morphology of non-uniform ellipse-like $\mathrm{nCaCO}_{3}$ particles[1,3,8,15,16,19,22]. 

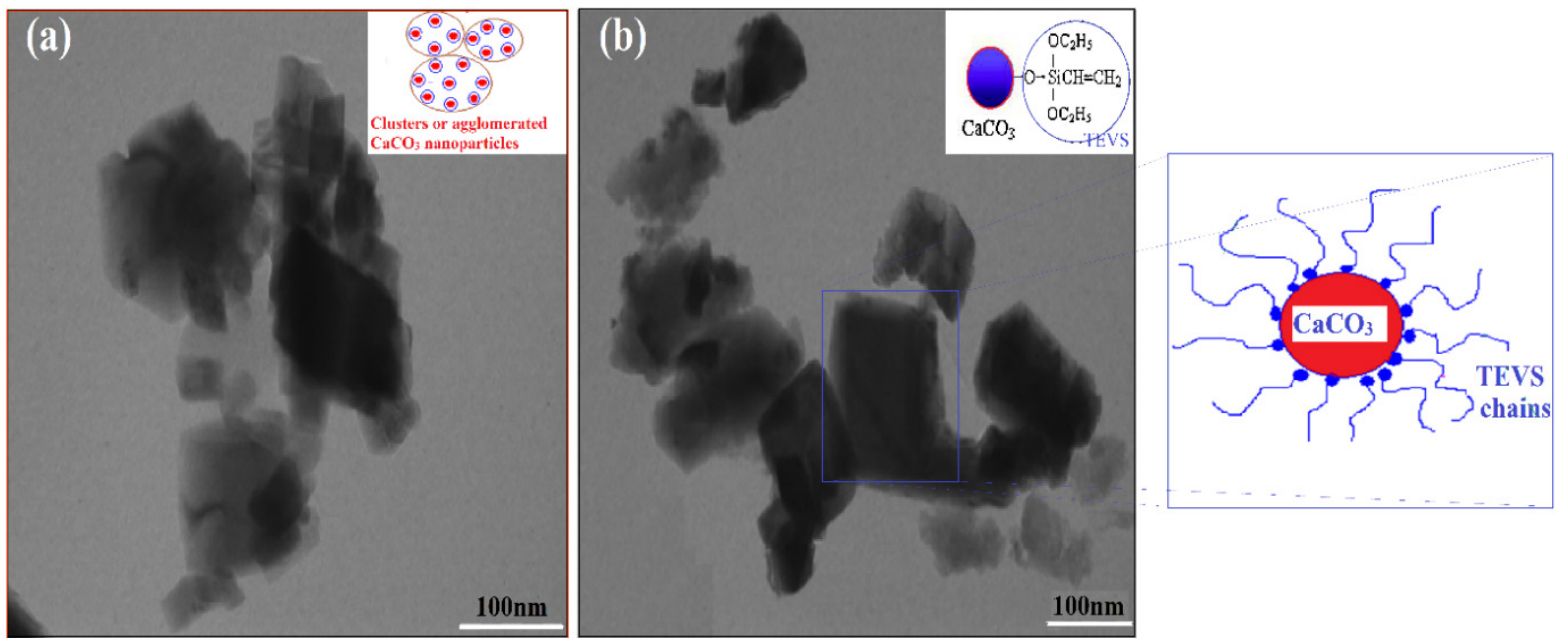

Figure 4. TEM of (a) unmodified $\mathrm{nCaCO}_{3}$ particles (b) surface modified $\mathrm{nCaCO}_{3}$ particles by TEVS

The agglomeration in untreated $\mathrm{CaCO}_{3}$ nanoparticles is due to electrostatic forces of attraction and therefore the $\mathrm{CaCO}_{3}$ nanoparticle behaves like a commercial $\mathrm{CaCO}_{3}[16]$. The increase in particle size of modified $\mathrm{nCaCO}_{3}$ particles i.e. $\sim 90 \mathrm{~nm}$ was due to its encapsulation by silane group of TEVS. The de-agglomeration of modified $\mathrm{CaCO}_{3}$ nanoparticles was due to surface modification, which reduces the surface energy along with reduction in electrostatic forces of attraction. A single $\mathrm{CaCO}_{3}$ nanoparticle can be observed in Figure 4(b) which looks like crystal structure and cubic morphology [11,14] due to ultrasound cavitation effect.

\subsection{Characteristic Properties of Modified and Unmodified $\mathrm{CaCO}_{3}$ Nanoparticles}

Nanoparticles of $\mathrm{nCaCO}_{3}$ particles $<39 \mathrm{~nm}$ (prepared by insitu deposition) can absorb $139 \mathrm{j} / \mathrm{g}$ of heat [1] and onset decomposition temperature was only $24.7^{\circ} \mathrm{C}$ [10]. It is reported that there was no change in onset decomposition temperature of TEVS modified $\mathrm{nCaCO}_{3}$, and percentage weight loss was $1.2 \%$ because of decomposition of silane coupling agent and adsorbed water on the particles surface [2]. Maximum weight loss (3-5\%) was occurred in the temperature range of $200-620^{\circ} \mathrm{C}$ due to thermal decomposition of modified nano- $\mathrm{CaCO}_{3}$ particles using of sodium stearate, dihydrogen phosphate and oleic acid [11].Unmodified $\mathrm{nCaCO}_{3}$ particles were reported with highest thermal stability with $3 \%$ weight loss during $0-430^{\circ} \mathrm{C}$ [14], which is also a characteristic property of $\mathrm{nCaCO}_{3}$ particles.

The effect of the surface modification can be evaluated by the relative contact angle and active ratio $[11,22]$. The water contact angle was observed at $122.25^{\circ}$ for the modified $\mathrm{nCaCO}_{3}$ in the presence of sodium stearate, dihydrogen phosphate and oleic acid [11]. No stable drop shape was observed, and water was imbibed in the pellet. The water contact angle of on pure $\mathrm{CaCO}_{3}$ powders was very small (nearly 0 ) and the unmodified $\mathrm{CaCO}_{3}$ is hydrophilic. Addingoleic acid to the $\mathrm{CaCO}_{3}$ processing, the relative contact angle was increased to $95^{\circ}$, indicated good hydrophobic character. The contact angle of the final modified $\mathrm{CaCO}_{3}$ using silane coupling agent was $152^{\circ}$, indicated super hydrophobic character [22]. The water contact angles of the pellets after drying at $100^{\circ} \mathrm{C}$ for $24 \mathrm{~h}$ were not changed, which indicated that volatile compounds were absent.The active ratio can reflect the effect of surface modification, which is defined as the weight ratio of the floated product to the overall product mixed with water under vigorous stirring and it can be evaluated by the floating test. It was observed that the amount of surfactant had a dramatic effect on the active ratio of $\mathrm{CaCO}_{3}$ nanoparticles. As the loading of modifiers or surfactants was increased, the active ratio of hydrophobic $\mathrm{CaCO}_{3}$ increased gradually and reached almost $99.9 \%$ when the loadings of modifiers to $\mathrm{CaCO}_{3}$ was only $2 \%$ and the product changed from hydrophilic to hydrophobic completely. However, when the dosage of modifiers was more than $2 \%$, part of the polar groups was outward owing to the multilayer physical adsorption. Whiteness value of $\mathrm{nCaCO}_{3}$ particles can be detected by digital displaying whiteness meter. The influence of the different modifiers loading on the value of whiteness showed that value of whiteness was increased as increase in modifier loadings[11].

XRD studies of modified and unmodified of $\mathrm{nCaCO}_{3}$ particles showed that ion binding, lattice matching and stereo chemical recognition were important factors for oriented nucleation. In the reaction of $\mathrm{CaCO}_{3}$ synthesis, $\mathrm{Ca}^{2+}$ was located at lattice position in (001) layers, alternating with layers of $\mathrm{CO}_{3}{ }^{2-} \cdot \mathrm{Ca}^{2+}$ can be bonded to the negatively charged surfactants and result in the nucleation of the modified $\mathrm{CaCO}_{3}$ [11].In XRD study, there was sharp peak observed at $2.5^{\circ}$ for $\mathrm{nCaCO}_{3}$ which was $2.8^{\circ}$ in the case of commercial $\mathrm{CaCO}_{3}[3]$.

\section{Conclusions}

Synthesis of $\mathrm{CaCO}_{3}$ nanoparticles and surface modification by TEVS were done successfully using optimized process of ultrasonic cavitation technique. During 
the synthesis and surface modification of $\mathrm{nCaCO}_{3}$ the major affecting process parameters were (i) lower reaction temperature (ii) faster conversion rate (iii) achieved higher conversion (iv) nanoparticles with well reduced size and (v) de-agglomeration and uniform distribution of nanoparticles. The morphology transformation is observed from growth and shape of the modified $\mathrm{nCaCO}_{3}$ particles due to the addition of surfactants. Therefore, a conclusion can be drawn that the compatibility with surfactants and the distribution of $\mathrm{nCaCO}_{3}$ particles are obviously enhanced, which are potential for the industrial process of biomineralization. This mineral inorganic $\left(\mathrm{CaCO}_{3}\right)$ nanomaterial can be used as a nanofiller as well as binder in the polymer and in paint industry. Surface modified $\mathrm{nCaCO}_{3}$ particles can show good dispersion in to the polymer matrix as compared to unmodified and commercially available material. Ultra-sonication technique is less time consuming with high yieldand uniform distribution. This method can also been applicable to the surface modification of other mineral nanofillers, which can be synthesized using chemical and/or physical route.

\section{REFERENCES}

[1] S. Mishra, S.H. Sonawane, R.P. Singh, J. Polym. Sci. B: Polym. Phy, 43(1), 107-113 (2005).

[2] S. Mishra, A. Chatterjee, R.P. Singh, Polym. Adv. Technol., 22(12), 2571-2582 (2011).

[3] S. Mishra, N.G. Shimpi, J. Verma, Rubberchem-2006 (Munich, Germany), 1-8 (2006).

[4] Z. Demjn, B. Puknszky, E. Fldes, J. Nagy, J. Colloid Interface Sci., 190(2), 427-436 (1997).

[5] S. Mishra, S.H. Sonawane, V. Chitodkar, Polym. Plast. Technol. Eng., 44(3), 463-473 (2005).

[6] N.G. Shimpi, R.U. Kakade, S.S. Sonawane, A.D. Mali, S. Mishra, Polym. Plast. Technol. Eng., 50(8), 758-761 (2011).

[7] N.G. Shimpi, S. Mishra, Polym. Plast. Technol. Eng., 51(2),
111-115 (2012).

[8] S.S. Sonawane, S. Mishra, N.G. Shimpi, Polym. Plast. Technol. Eng., 4(1), 38-44 (2009).

[9] N.G. Shimpi, J. Verma, S. Mishra, J. Comp. Mater., 44(2), 211-219 (2011).

[10] S. Mishra, N.G. Shimpi, J. Sci. Ind. Res., 64(10), 744-751 (2005).

[11] Z.L. Na, F.J. Dong, W.Z. Chen, Sci. China. Ser. B-Chem., 52(7), 924-929 (2009).

[12] S. Mishra, A. Mukherji, Polym. Plast. Technol. Eng., 46(3), 239-244 (2007).

[13] Y. Sheng, J. Zhao, B. Zhou, X. Ding, Y. Deng, Z. Wang, Mater. Lett., 60(27), 3248-3250 (2006).

[14] S.P. Gumfekar, K.J. Kunte, L. Ramjee, K.H. Kate, S.H. Sonawane, Prog. Org. Coat., 72(4), 632- 637 (2011).

[15] S. Mishra, N.G. Shimpi, U.D. Patil, J. of Polym. Res., 14(6), 449-459(2007).

[16] S. Mishra, N.G. Shimpi, A.D. Mali, Polym. Adv. Technol., 23(2), 236-246 (2012).

[17] S. Mishra, N.G. Shimpi, A.D. Mali, J. Polym. Res., 18(6), 1715-1724 (2011).

[18] S. Mishra, U.D. Patil, N.G. Shimpi, Polym. Plast. Technol. Eng., 48 (10), 1078-1083 (2009).

[19] N.G. Shimpi,S. Mishra, J. Nanopart. Res., 12(6), 2093-2099 (2010).

[20] A.G. Xyla, P.G. Koutsoukos, J. Chem. Soc., Faraday Trans. 1, 183(5), 1477-1484 (1987).

[21] K. Premphet, P. Horanont, Polym., 41(26), 9283-9290 (2000).

[22] C. Wang, C. Piao, X. Zhai, F.N. Hickman, J. Li, Powd. Technol., 200(1-2), 84-86 (2010).

[23] J.M. Didymus, P. Oliver, S. Mann, A.L. Devires, P.V. Hauschka, P. Westbroek, J. Chem. Soc., Faraday Trans., 89(15), 2891-2900 (1993).

[24] T. Kato, A. Sugawara, N. Hososada, Adv. Mater., 14(12), 869-877 (2002). 